\title{
Virchow's Node Not Always Signifies a Primary Gut or Lung Malignancy. Look Further and Farther
}

\author{
A. L. Hemalatha, Nishtha Batra, Deepthi. B. Ramesh and S. D. Shashikumar \\ Department of Pathology, Mysore Medical College and Research Institute, Mysore, Karnataka, India \\ Correspondence should be addressed to: A. L. Hemalatha; halingappa@gmail.com
}

Received 11 June 2013; Accepted 4 July 2013; Published 28 August 2013

Academic Editor: Akishi Ooi

Copyright (C A. L. Hemalatha, Nishtha Batra, Deepthi. B. Ramesh and S. D. Shashikumar. Distributed under Creative Commons CC-BY 3.0

\begin{abstract}
Virchow's node generally signifies metastasis from primary malignancy either in gastrointestinal tract or lung. Apart from the commonly recognized primary sites, $4.5-5 \%$ of primary testicular cancers are also known to metastasize to the left supraclavicular lymph node. Amidst the time and space restraint of a busy FNAC practice, one may tend to overlook considering testis as one of the possible primary sites for malignancy while aspirating a left supraclavicular lymph node and interpreting the smears. This case report throws light on the importance of considering primary germ cell tumor of the testis as one of the differential diagnoses, while aspirating a Virchow's lymph node, especially in a young male patient, besides emphasizing the need for a thorough clinical examination.
\end{abstract}

Keywords: Virchow's node; Metastasis; Testicular tumour.

\section{Introduction}

Presence of Virchow`s nodes generally alerts the clinician and the pathologist alike to suspect and investigate for the primary in the gastro-intestinal tract or lung malignancy. Other than these sites, 4.5\%$5 \%$ of primary testicular cancers have been reported to have neck metastasis ${ }^{2}$. Neck metastasis from germ cell tumors are relatively rare. Therefore, the clinicians and the pathologists may overlook Metatstatic Germ cell tumor under the differential diagnoses for left supraclavicular lymphadenopathy ${ }^{6}$. But, the possibility of neck metastasis from a primary testicular cancer, in a young male patient, has to be borne in mind always.

The present case report highlights this importance and reinforces the need to include metastatic germ cell tumors in the differential diagnosis of left supraclavicular lymphadenopathy. Besides this, it also stresses on the necessity for thorough history elicitation and detailed clinical examination, however impractical it may seem in a busy FNAC clinic.

\section{Case Report}

A 24 year old male patient presented with the history of a swelling in the left side of

Cite this Article as: A. L. Hemalatha, Nishtha Batra, Deepthi. B. Ramesh and S. D. Shashikumar (2013), "Virchow's Node Not Always Signifies a Primary Gut or Lung Malignancy. Look Further and Farther," International Journal of Case Reports in Medicine, Vol. 2013 (2013), Article ID 616832, DOI: $10.5171 / 2013.616832$ 
the neck, which was noticed 10 days earlier. Local examination showed a single, enlarged, left supraclavicular lymph node measuring $4 \times 3 \mathrm{~cm}$, with a firm to hard consistency.

FNAC from the lymph node yielded moderately cellular smears, with pleomorphic tumor cells in clusters, singles and acinar patterns. The cells showed moderate amounts of eosinophilic cytoplasm, high nucleo-cytoplasmic ratio, irregular nuclear borders and prominent nucleoli. Focal areas showed a myxoid background. (Fig-1, 2)

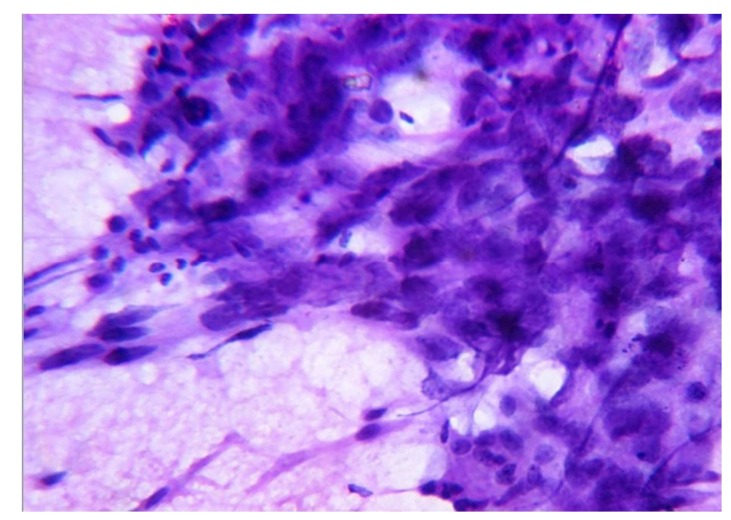

Fig 1 - FNAC Smear from the Left Supraclavicular Lymph Node - H\&E (40x) - Pleomorphic Tumor Cells in Clusters, Singles and Acinar Pattern with High Nucleo-Cytoplasmic Ratio Irregular Nuclear Borders, Prominent Nucleoli and Moderate Amount of Eosinophilic Cytoplasm

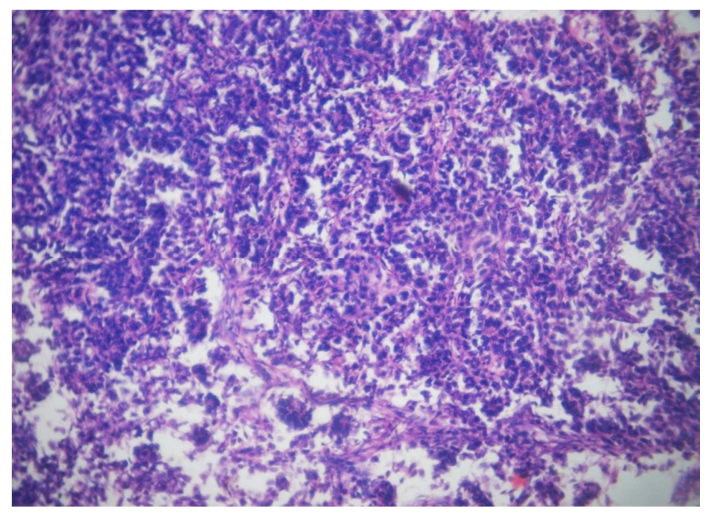

Fig 2 - H\&E (10x) - Histopathological Section from the Primary Testicular Germ Cell Tumor with Features of Embryonal Carcinoma which was a Minor Component

With these features, a cytological diagnosis of metastatic deposits from a poorly differentiated carcinoma was arrived at. Detailed history elicitation, thorough clinical examination and review of patient records were undertaken to search for a clue about the primary site of malignancy.
Review of records revealed a surprise element, in the history of high orchidectomy performed six months earlier for a testicular tumor. Review of the histopathology slides showed features of mixed germ cell tumor with major components of Teratoma (Fig-3) and Yolk sac tumor (Fig-4), and a minor component of Embryonal carcinoma (Fig-2). 


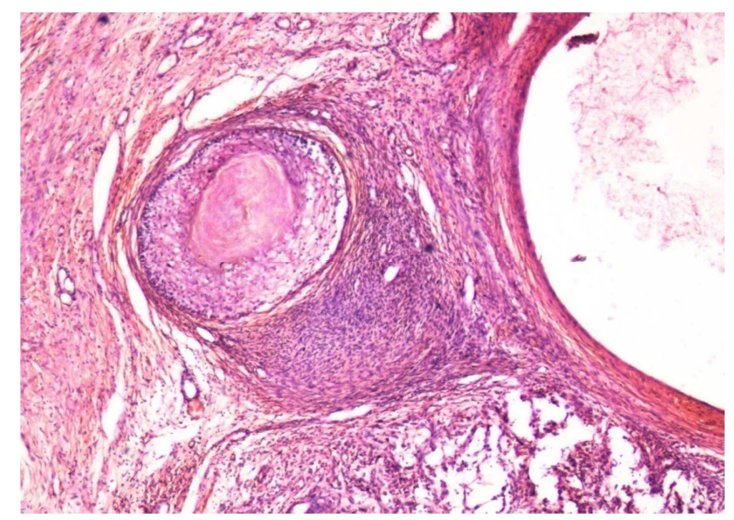

Fig 3 - H\&E (10x) - Histopathological Section from the Primary Testicular Germ Cell Tumor with Features of Teratoma which was a Major Component

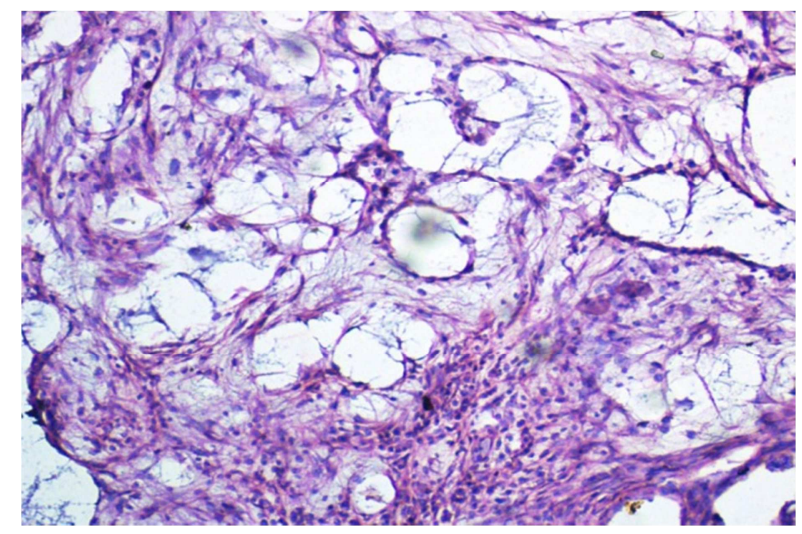

\section{Fig 4 - H\&E (10x) - Histopathological Section from the Primary Testicular Germ Cell Tumor with Features of Yolk Sac Tumor which was a Minor Component}

Final Diagnosis - Metastasis in the left supraclavicular lymph node from the Embryonal component of Mixed Germ Cell tumour of the testis.

\section{Discussion}

Neck metastasis from primary testicular malignancies is rare, with a reported incidence of $4-5 \% .{ }^{3}$ Involvement of the cervical lymph nodes indicates advanced disease.

The cytological features of embryonal carcinoma are less specific and indistinguishable from poorly differentiated malignancies such as high grade carcinoma ${ }^{1}$. This explains why the diagnosis of metastasis from poorly differentiated carcinoma was initially considered in the present case. An index of suspicion to consider mixed germ cell tumors, especially those with a component of embryonal carcinoma, is always required in the diagnosis of these tumors ${ }^{4}$.

The young age of the patient is the other important pointer towards Germ cell tumors $^{6}$. Metastasis from Germ cell tumors occurs in young patients and carcinomas tend to affect elderly patients.

There has been a remarkable advance in the treatment of germ cell tumors and most patients with germ cell tumors, even those with disseminated disease are potentially curable and respond to modern chemotherapy4.

The treatment of metastatic carcinomas is generally palliative and the prognosis is dismal. So, a missed diagnosis of metastatic 
germ cell tumor has serious implications in that, a tumor with a better prognosis is mistaken for the one with a worse prognosis. Hence, it is crucial for the pathologist to be able to consider metastasis from primary germ cell tumor and alert the clinician to such a probability 4 . This emphasizes the fact that, while dealing with neck node metastasis in a male, the possibility of metastatic germ cell tumor should also be considered along with the other commonly known differential diagnoses 5 .

\section{Conclusion}

The present study bears a message for the cytologist, to pay due attention to the valuable clues which lie within proper history elicitation and thorough clinical examination, for, the cytological smears alone, don't say it all.

\section{References}

1. Highman, W. J. \& Oliver, R. T. (1987). "Diagnosis of Metastases from Testicular Germ Cell Tumours Using Fine Needle Aspiration Cytology," Journal of Clinical Pathology, 40 (11) 1324-1333.

2. Jemal, A., Siegel, R., Ward, E., Murray, T., Xu, J., Smigal, C. \& Thun, M. J. (2006). "Cancer Statistics, 2006," CA: A Cancer Journal for Clinicians, 56 (2) 106-130.

3. Mustafa, A., Schwentner, I., Schmutzhard, J., Strasser, H. \& Sprinzl, G. M. (2009). "Neck Metastasis of the Testicular Teratoma in an Adult: a Case Report," Brazilian Journal of Otorhinolaryngology, 75 (5) 766-766.

4. Shek, T. W., Yuen, S. T., Luk, I. S. \& Wong, M. P. (1996). "Germ Cell Tumour as a Diagnostic Pitfall of Metastatic Carcinoma," Journal of Clinical Pathology, 49 (3) 223-225.

5. Tamboli, S., Agashe, S. \& Patil, P. (2012). "Cytologic Diagnosis of Metastatic Seminoma in Neck Nodes as Initial Presentation," JKIMSU, 1 (1) 99101.
6. True, L. D., Rosai, J., Tumors of Testis.In: Mc Gee JO`G, Issacson PG, Wright N A,Eds (1992). 'Oxford Textbook of Pathology,' Oxford: Oxford University Press, 1554-62. 